\title{
Encapsulation of a gold nanoparticle in a DNA origami container
}

\author{
Akinori Kuzuya $^{1,2}$, Masafumi Kaino ${ }^{1}$, Mirai Hashizume ${ }^{1}$, Kazuki Matsumoto ${ }^{1}$, Takeaki Uehara ${ }^{1}$, \\ Yasutaka Matsuo ${ }^{3}$, Hideyuki Mitomo ${ }^{3}$, Kenichi Niikura ${ }^{3}$, Kuniharu Ijiro ${ }^{3}$ and Yuichi Ohya ${ }^{1}$
}

A 'box-shaped' three-dimensional (3D) DNA origami of $\sim 40-\mathrm{nm}$ dimensions was selectively formed by closing a symmetric open motif with three orthogonal faces. This 3D DNA origami was used as an intelligent nano-container to encapsulate exactly one 10-nm gold nanoparticle (AuNP). AuNPs were functionalized with thiol-modified DNA strands and attached to one of the faces of the open motif, which was designed to be an interior surface of the box and decorated with three complementary strands. The open motif was then closed into the box shape as triggered by the addition of DNA strands joining the remaining edges. An examination of the suitable folding path of an M13 scaffold using fluorescently labeled staple strands revealed that the flexibility at the hinge was essential for the efficient closing of the DNA origami container. Atomic force microscope and transmission electron microscope imaging of agarose-gel-purified complexes clearly showed the successful encapsulation of one AuNP inside the shell.

Polymer Journal (2015) 47, 177-182; doi:10.1038/pj.2014.128; published online 17 December 2014

\section{INTRODUCTION}

The field of 'fusion materials,' such as organic and inorganic materials, bio and non-bio hybrids and molecularly self-assembled biomimetic materials, is attracting more researchers in not only the material sciences but also in the biomedical and nano sciences. These materials are produced by fabrication processes with low energy costs and are expected to exhibit distinctive structural and functional properties superior to conventional materials and natural biological systems. ${ }^{1,2}$

Gold nanoparticle (AuNP)-DNA hybrids are typical fusion materials that have been popularly studied to date ${ }^{3}$ because of the established conjugation technology between AuNPs and DNA. ${ }^{4-7}$ As a result, large pools of various two-dimensional and three-dimensional (3D) DNA nanostructures have been developed from structural DNA nanotechnology based on branched DNA motifs. ${ }^{8}$ Among the 3D DNA nanostructures, DNA origami,${ }^{9}$ in which long single-stranded DNA scaffolds are folded into designed nanostructures with the aid of many short staple strands, provides one of the most precise arrangement of AuNP at nanometer resolutions. These AuNP-DNA arrays are typically two-dimensional except for a few 3D origami comlexes. ${ }^{10-20}$ Although AuNP-DNA origami cage complexes have been elegantly constructed by Yan et al. ${ }^{17}$ no complex in which AuNP is completely covered with DNA origami has been reported.

We have recently constructed a 'box-shaped' 3D DNA origami structure of $44 \times 42 \times 35 \mathrm{~nm}$ dimensions ${ }^{21}$ along with other hollow structures consisting of planar faces. ${ }^{22-25}$ Our 3D DNA origami has two distinguishing design features. The first feature is that controlled right angles between the faces are achieved by selecting appropriate positions for crossovers connecting the faces. Typically, the crossovers in DNA origami are placed every $16 \mathrm{bp}$ to connect DNA helices at angles of $180^{\circ}$. In our box design, the number of nucleotides between the crossovers at the edges of the faces is intentionally reduced to $8 \mathrm{bp}$, which corresponds to 0.76 helical turns. Thus, the dihedral angle between the two faces next to an edge is uniformly fixed at $90^{\circ}$ in a predetermined direction. Due to this strategy, the side of the DNA sheet that faces the inside of the box and the side that faces the outside are fully controlled. The second feature is a two-step folding mechanism (Scheme 1). We designed the box to fold first into an open form composed of two units, each of which is made of three orthogonally connected faces (that is, open motif (OM)). The complex then closes into a box shape using short helper strands to connect the three edges of the two units (closed motif $(\mathrm{CM})$ ). Accordingly, our 3D DNA origami are one of the earliest DNA origami structures that feature mechanical movement. ${ }^{26}$ Both of these aforementioned features are incorporated for the purposes of guest nanomaterial encapsulation.

In this study, we applied our 3D DNA origami as a nano-container for the first time, to selectively encapsulate exactly one inorganic nanoparticle inside a shell. A 10-nm AuNP functionalized with thiolmodified DNA strands was attached to one of the faces of an OM DNA origami container. The complex was then closed into a box shape triggered by the addition of the helper strands joining the remaining edges. A suitable folding path of an M13 scaffold designed for an efficient closing was also examined using fluorescently labeled staple strands.

\footnotetext{
${ }^{1}$ Department of Chemistry and Materials Engineering, Kansai University, Suita, Osaka, Japan; ${ }^{2}$ PRESTO, Japan Science and Technology Agency, Kawaguchi, Saitama, Japan and ${ }^{3}$ Research Institute for Electronic Science, Hokkaido University, Kita-Ward, Sapporo, Japan

Correspondence: Dr A Kuzuya or Professor Y Ohya, Department of Chemistry and Materials Engineering, Kansai University, 3-3-35 Yamate, Suita, Osaka 564-8680, Japan. E-mail: kuzuya@kansai-u.ac.jp or yohya@kansai-u.ac.jp

Received 30 July 2014; revised 6 November 2014; accepted 18 November 2014; published online 17 December 2014
} 

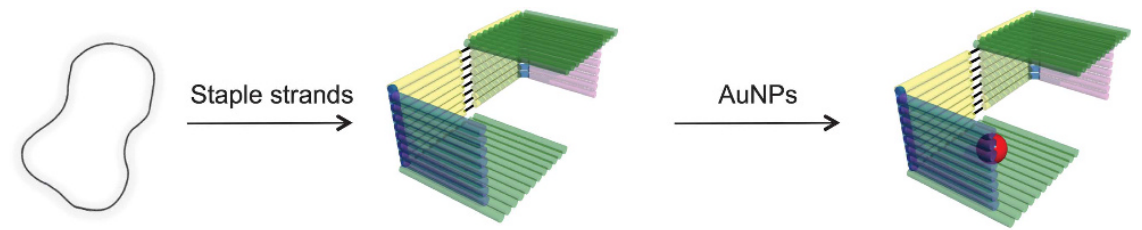

M13 scaffold

Open motif (OM) without hinge strands (OM")
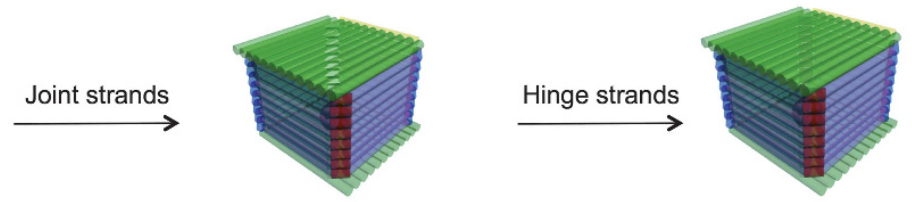

Closed motif (CM) without hinge strands (CM")

$\mathrm{CM}$

Scheme 1 Selective gold nanoparticle (AuNP) capture into the DNA origami container.

\section{EXPERIMENTAL PROCEDURE}

Standard desalted grade staple and helper strands (Supplementary Table S1) were purchased and used without further purification from Integrated DNA Technologies (Coralville, IA, USA) and Sigma Genosys (Ishikari, Japan). Highperformance liquid chromatography-purified, fluorescently labeled strands were purchased from Sigma Genosys. The M13mp18 single-stranded DNA (Takara, Japan) was used for the DNA origami scaffold. Bis(p-sulfonatophenyl) phenylphosphine-coated AuNPs were prepared as described in a previous study. ${ }^{27}$

The formation of DNA origami container in the open form (that is, OM) was performed with M13mp18 single-stranded DNA (4 nM) and staple strands ( $20 \mathrm{nM}$ for each strand) in a solution containing Tris $(40 \mathrm{~mm})$, acetic acid $(20 \mathrm{~mm}), \operatorname{EDTA}(10 \mathrm{~mm})$ and magnesium acetate $\left(12.5 \mathrm{~mm}, 1 \times \mathrm{TAE} / \mathrm{Mg}^{2+}\right.$ buffer, $50 \mu \mathrm{l})$. This mixture was cooled from $90^{\circ} \mathrm{C}$ to $25^{\circ} \mathrm{C}$ at a rate of $-1.0^{\circ}$ $\mathrm{C} \min ^{-1}$ using a PCR thermal cycler (Bio-Rad T100, Hercules, CA, USA) to anneal the strands.

The selective closing of the OM into the CM was achieved by adding 5 eq. of helper strands stepwise to the solution of the OM. For instance, $4 \mathrm{~nm} \mathrm{OM}$ annealed in $1 \times \mathrm{TAE} / \mathrm{Mg}^{2+}$ buffer $(20 \mu \mathrm{l})$ was combined with $2.5 \mu \mathrm{l}$ of a $400-\mathrm{nM}$ mixture of 14 helper strands to first join three open edges. The mixture underwent mild annealing from 37 to $25^{\circ} \mathrm{C}$ for five cycles at a rate of $-1.0^{\circ}$ $\mathrm{C} \min ^{-1}$ using a PCR thermal cycler. Staple strands complementary to the hinge region were then added to the solution to form a fully hybridized CM after additional mild annealing.

Fluorescence measurements were made using an FP-6500 spectrometer (JASCO, Hachioji, Japan). Samples of DNA origami solutions (5 nM) were measured in $1 \times \mathrm{TAE} / \mathrm{Mg}^{2+}$ buffer $(50 \mu \mathrm{l})$ in the presence of 1.2 eq. fluorescent strands at an excitation wavelength of $450 \mathrm{~nm}$.

Atomic force microscope (AFM) imaging of DNA origami was performed on a Multimode 8/Nanoscope V system (Bruker AXS, Billerica, MA, USA). The DNA origami solution $(1 \mu \mathrm{l})$ was deposited on a freshly cleaved mica substrate, additional $1 \times \mathrm{TAE} / \mathrm{Mg}^{2+}$ buffer $(40 \mu \mathrm{l})$ was added and the imaging was performed under the fluid PeakForce Tapping mode with a BL-AC40TS tip (Olympus, Shinjuku, Japan).

The DNA functionalization of 10-nm AuNPs was achieved by adding $300 \mu \mathrm{m}$ of thiol-modified 24-mer DNA (5'-HS-TTTTTTGTTGTTGTTGTTGTTGTT $\left.-3^{\prime}, 50 \mu \mathrm{l}\right)$ to the $12.4-\mu \mathrm{M} \operatorname{Bis}(p$-sulfonatophenyl)phenylphosphine-coated AuNP dispersion $(12.1 \mu \mathrm{l})$ in the presence of $50 \mathrm{~mm} \mathrm{NaCl}$ and kept at room temperature overnight. Excess DNA was removed using an Amicon Ultra $100 \mathrm{~K}$ centrifugal filter (Merck Millipore, Billerica, MA, USA), and the functionalized AuNPs were re-dispersed in $1 \times \mathrm{TAE} / \mathrm{Mg}^{2+}$ buffer. Control AuNPs were accordingly prepared using a thiol-modified 24-mer oligo-T DNA (5'-HS-TTTTTTTTTTTTTTTTTTTTTTTT- $\left.{ }^{\prime}\right)$. The attachment of DNAfunctionalized AuNPs to the $\mathrm{OM}^{\prime \prime}$ DNA origami was achieved by adding 10 eq. AuNPs $(6.5 \mu \mathrm{l})$ to the solution of $10 \mathrm{~nm} \mathrm{OM}{ }^{\prime \prime}(500 \mu \mathrm{l})$ prepared with $1.2 \mathrm{eq}$. AuNP-binding strands for five cycles of mild annealing from 37 to $25^{\circ} \mathrm{C}$ at a rate of $-1.0^{\circ} \mathrm{C} \mathrm{min}^{-1}$. Subsequently, the AuNP-complexed $\mathrm{OM}^{\prime \prime}$ was treated as described above to convert into a $\mathrm{CM}$.

Agarose gel electrophoresis was carried out in a water bath using $1.5 \%$ agarose gel containing $1 \times \mathrm{TAE} / \mathrm{Mg}^{2+}$ buffer at $200 \mathrm{~V}$ for $1.5 \mathrm{~h}$. The gel was stained with GelStar (FMC Bioproducts, Philadelphia, PA, USA) and imaged on an ImageQuant LAS 4000 equipped with the Epi-BGR Set (GE Healthcare, Buckinghamshire, UK). The band corresponding to the CM-AuNP complex was cut out, and the complex was extracted using Freeze'N Squeeze DNA gel extraction spin columns (Bio-Rad).

For transmission electron microscope imaging, collodion-coated grids were treated with $5 \mu \mathrm{l}$ of the extracted solution of the complex for $40 \mathrm{~min}$ at room temperature. The samples were then positively stained for 3 min with an EM Stainer solution (Nisshin EM, Shinjuku, Japan) prior to the imaging on a JEM-1400 microscope (JEOL, Akishima, Japan) at $80-\mathrm{kV}$ acceleration voltage.

\section{RESULTS AND DISCUSSION}

Figure 1a shows the original design of the DNA origami container reported in the previous study (see Supplementary Figure S1 for details). ${ }^{25}$ In total, 200 staple strands (12-40 nucleotides; green, violet and yellow series) were used to fold a circular, 7249-nucleotide, singlestranded M13mp18 genome into a complex of two squares of 120 $\mathrm{bp} \times 12$ helices (green faces, ca. $40 \times 42 \mathrm{~nm}$ ) and four rectangles of $96 \mathrm{bp} \times 8$ helices (violet and yellow faces, ca. $32 \times 28 \mathrm{~nm}$ ) in a C2 symmetry (OM in Scheme 1). The violet-yellow combinations were connected to each other by M13 scaffolds (black lines) and 24nucleotide (nt) joint strands (drawn in blue). The yellow-yellow combination indicated by an arrow was similarly connected by the scaffold and the hinge strands (in maroon). The phage scaffold adjacent to these joint edges was kept single-stranded and 4-nt long for both of the sides to introduce flexibility around the edges (thin black lines). In the helices between the violet and green faces, the number of nucleotides between the crossover junctions that connected adjacent helices was reduced to $8 \mathrm{nt}$ (from the regular number of $16 \mathrm{nt}$ in the other helices) to make right angles between the two faces. Thus, both of the violet faces were oriented in the same direction from the green faces, that is, out of the plane of the paper toward the reader. Because the yellow and green faces were connected via the M13 scaffold at the opposite edges of the violet faces (black dotted lines), the green, violet and yellow faces formed a tetrahedral structure when annealed in the presence of the staple and blue-edge strands. The motifs formed in the absence of the red joint strands were in an open form $(\mathrm{OM})$, and turned into a closed form (CM) with the mild annealing of red joint strands as shown in Scheme 1. 
a

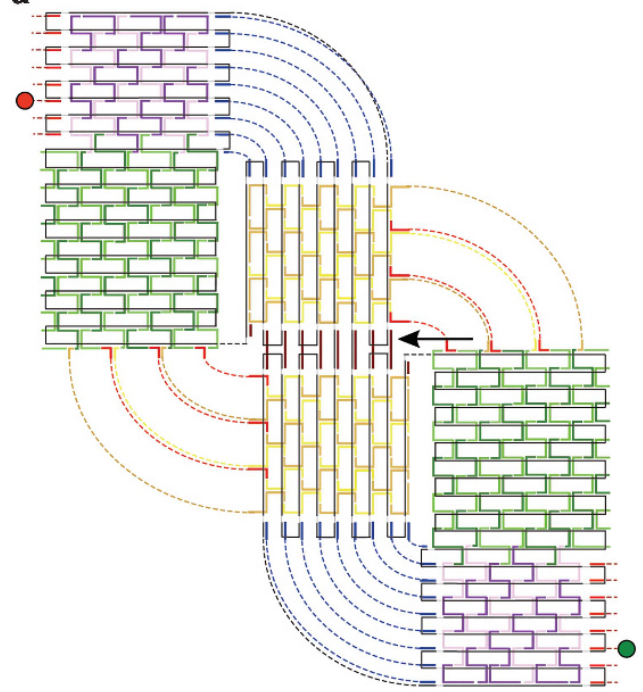

b

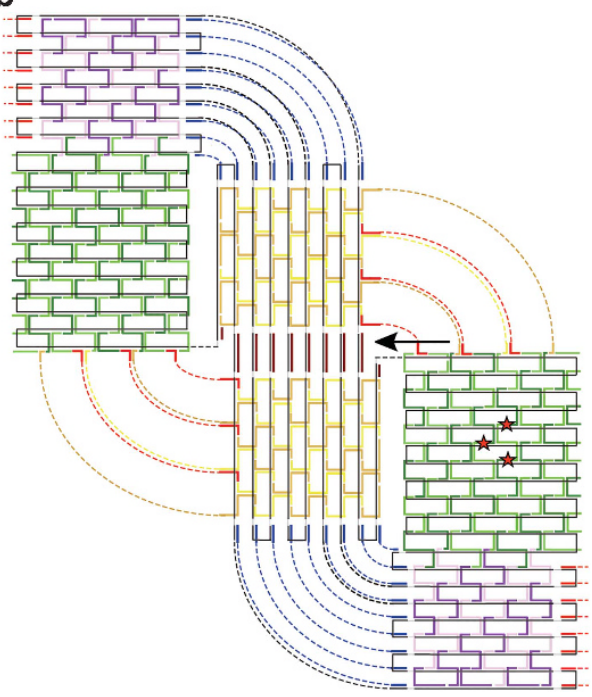

Figure 1 Design of the DNA origami container. (a) The original folding path of a single-stranded, circular M13mp18 phage genome (the black line) and hybridization pattern of the staple and the joint strands (colored lines). In total, 200 staple strands and 32 joint strands are used to fold the scaffold into six faces (yellow, green and violet faces) and four joint edges (blue, maroon and red edges). The dotted lines represent direct connections of the strands, and the thin portions in the black line represent single-stranded flexible regions introduced next to the joint edges. The original folding path contains only two continuous stretches of the scaffold at the hinge as indicated by arrows. Positions of fluorescent dyes attached to the joint strands are indicated by circles (6-fluorescein amide (FAM) in green and Texas Red (TEX) in red). (b) The revised folding path with fully continuous hinge. Connections between the yellow and violet faces are accordingly modified (black dotted lines). Strands at the hinge (10 strands drawn in maroon) are separately added to the solution after closing of OM triggered by the addition of joint strands (red strands). Positions of 24-mer complementary DNA extensions for AuNP attachment are indicated by the red stars.

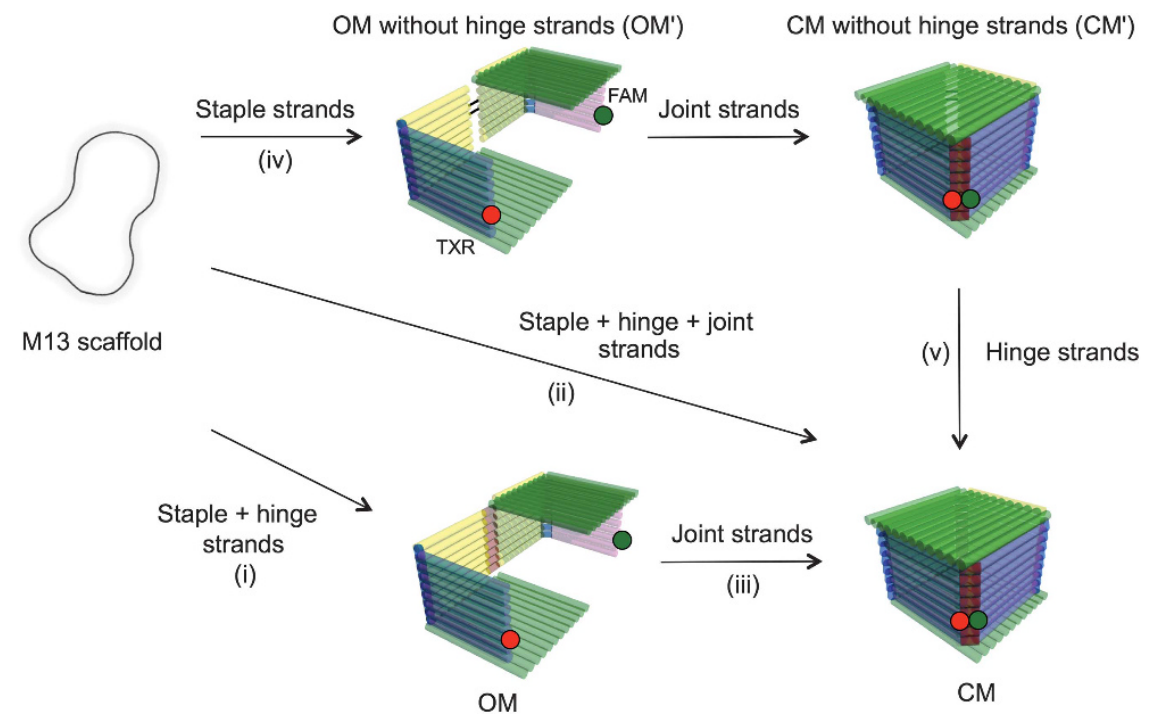

Scheme 2 Closure pathways of the DNA origami container. CM, closed motif; FAM, 6-fluorescein amide; TXR, Texas Red; OM, open motif.

Before attaching AuNPs to the DNA origami container, the yield of the structural change from $\mathrm{OM}$ to $\mathrm{CM}$ was estimated using fluorescently labeled motifs (Scheme 2). One of the joint strands on the red edge was split into two halves, and the two newly formed ends were modified with a pair of fluorescent dyes: 6-fluorescein amide and Texas Red (green and red circles, respectively, in Figure 1a). The excitation spectrum of Texas Red overlapped with the emission spectrum of 6-fluorescein amide. Thus, fluorescent resonance energy transfer occurred between the two dyes at close proximities. The emission spectra of the solutions of the OM and the CM prepared in various processes are shown in Figure 2. Compared with the spectrum of the OM formed in the presence of hinge strands (i), a significant quenching (ca. 24\%) of 6-fluorescein amide emissions was observed for the CM formed in two steps after adding joint strands to the OM solution (iii). This showed that the structural change induced in the shift from the $\mathrm{OM}$ to the $\mathrm{CM}$ brought the two dyes within a range sufficient for efficient fluorescent resonance energy transfer. However, the quenching yield remained lower than that for the CM directly formed from the M13 scaffold in one step $(\sim 28 \%)$ in the presence of all staple, joint and hinge strands (ii). Only $80 \%$ of the OMs closed 
into CMs when three of the edges (two edges in blue and the hinge in maroon) were hybridized in OM. When the hinge was kept single stranded in an $\mathrm{OM}\left(\mathrm{OM}^{\prime}\right.$, (iv)), 97\% of the $\mathrm{OM}^{\prime}$ turned into $\mathrm{CMs}$ after the addition of joint strands followed by mild annealing (to obtain the $\mathrm{CM}^{\prime}$ ) and the subsequent addition of hinge strands (v). The high yield of CMs prepared in the three-step $\mathrm{OM}^{\prime}-\mathrm{CM}^{\prime}-\mathrm{CM}$ process indicated the essential role of flexibility at the hinge in obtaining an efficient closing of an OM into a CM. However, this also highlighted the need to modify the DNA origami design. In the original design of the DNA origami container (Figure 1a), there were only two direct scaffold connections (black lines) between the two yellow faces at the

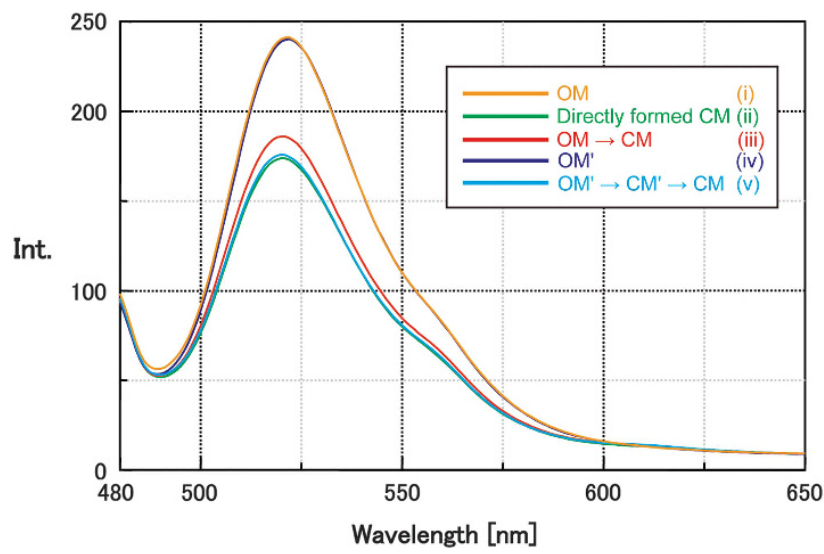

Figure 2 Fluorescent resonance energy transfer (FRET) assays to estimate the yields of selective structural change from open motif $(\mathrm{OM})$ to closed motif (CM). Emission spectra of 6-fluorescein amide (FAM) before and after the addition of the closure strands for the original folding pattern are shown. (i) $\mathrm{OM}$ formed in the presence of hinge strands (in orange). (ii) $\mathrm{CM}$ directly formed from M13 scaffold in one step in the presence of staple, joint and hinge strands (in green). (iii) CM formed in two steps by the addition of joint strands to $\mathrm{i}$ (in red). (iv) $\mathrm{OM}^{\prime}$ formed in the absence of hinge strands (in blue). (v) $\mathrm{CM}$ formed in three steps by the addition of joint strands to iv followed by mild annealing and addition of the hinge strands (in cyan). hinge region (indicated by the arrow). Consequently, the scaffold in the hinge must be hybridized to complementary hinge strands (drawn in maroon) in the $\mathrm{OM}$ to avoid undesired twists between the two symmetric units around the two connections in the $\mathrm{OM}^{\prime}$.

Thus, we revised the design of the DNA origami container as shown in Figure 1b. Here, the folding path of the M13 scaffold was altered to directly connect all eight helices composing the yellow faces via stretches of the scaffold at the hinge region (indicated by an arrow). This maintains the flexible single-stranded hinge in the $\mathrm{OM}^{\prime \prime}$, even in the absence of the complementary hinge strands (in maroon), while avoiding the undesired twist. The hinge strands can then be separately added to the system after the $\mathrm{CM}^{\prime \prime}$ is formed with the red joint strands first. Accordingly, the connections between the violet and yellow faces (dotted lines in black) were modified to accept the change.

The AFM imaging on mica of the $\mathrm{CM}$ in the revised design, prepared by the $\mathrm{OM}^{\prime \prime}-\mathrm{CM}^{\prime \prime}-\mathrm{CM}$ sequence, confirmed the successful formation of the expected 3D structures with fairly uniform shapes and heights comparable to the previous results $(6-8 \mathrm{~nm}$, Figure 3$)$. Few undesired OMs were observed, showing that the three-step process was well conserved with the revised design.

The encapsulation of a 10-nm AuNP was performed as illustrated in Scheme 1. Excess AuNPs were functionalized with 24-mer DNA strands and incubated in an $\mathrm{OM}$ in the revised design bearing complementary strands on one of the two green faces. Three staple strands in the center of a face were extended to tether the complementary strands in the same directions as the orientations of the violet faces (stars in Figure 1b). Thus, the attached AuNP was placed on the side that was designed to be the interior surface of the hollow CM. Subsequently, the OM-AuNP complex was treated with red joint strands, mildly annealed and fully closed with maroon hinge strands into a CM-AuNP complex.

To separate unbound AuNPs and to purify the CM-AuNP complexes, the annealed final mixture was subjected to electrophoresis in $1.5 \%$ agarose gel in $1 \times \mathrm{TAE} / \mathrm{Mg}^{2+}$ buffer (Figure $4 \mathrm{a}$ ). When the gel was photographed under white light illumination (Figure $4 \mathrm{a}$, left), a faint but significant band was observed that corresponded to the
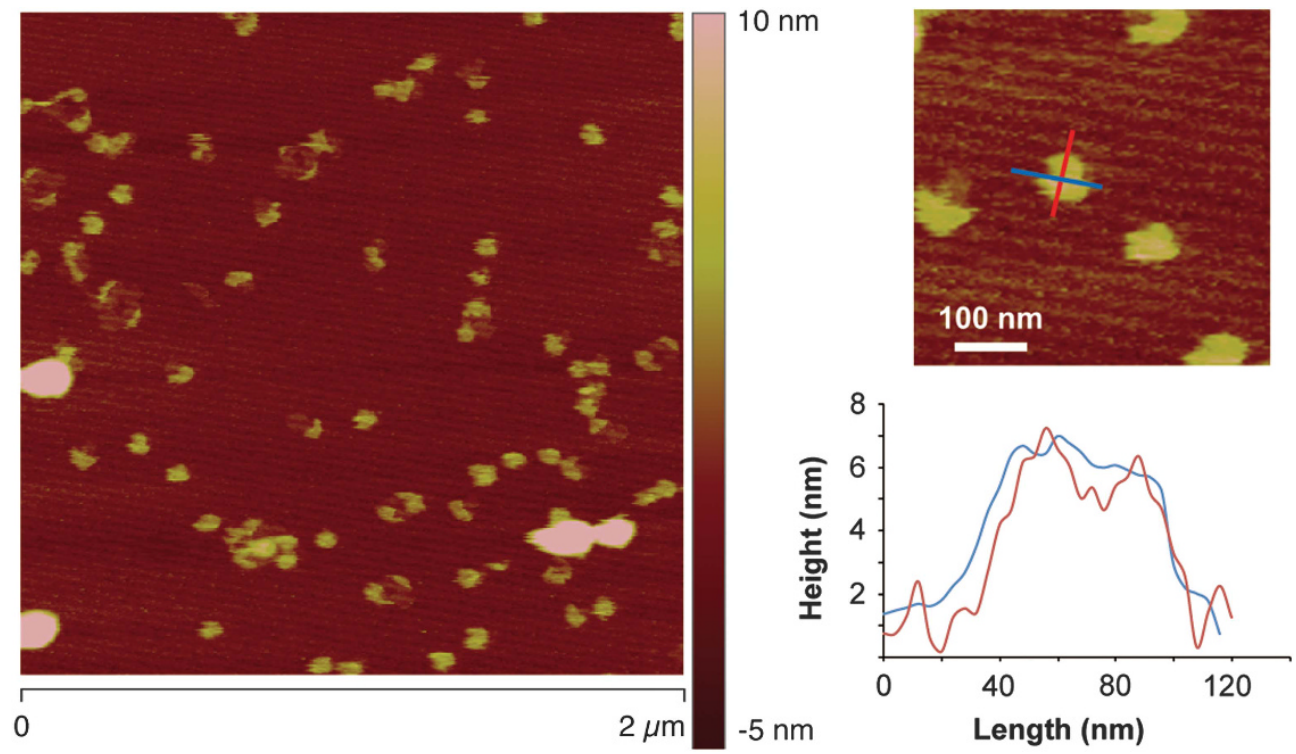

Figure 3 An atomic force microscope (AFM) image of closed motif (CM) with the revised folding pattern without gold nanoparticle (AuNP) attachment. The height profile of a typical motif is shown at the right. 
a

AuNPs Absorbance

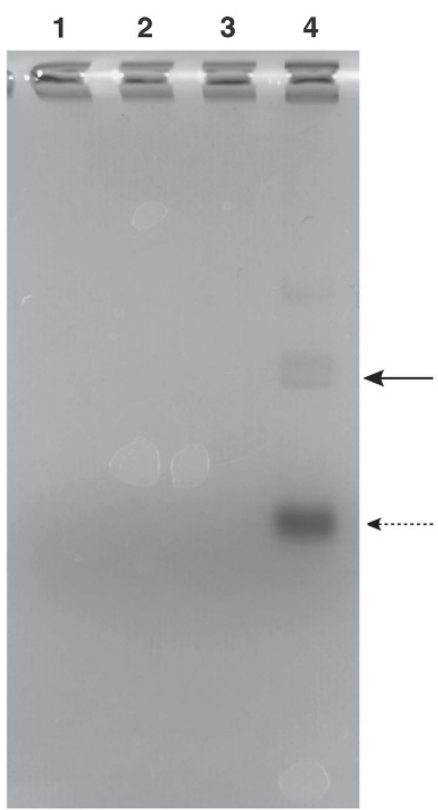

DNA Staining

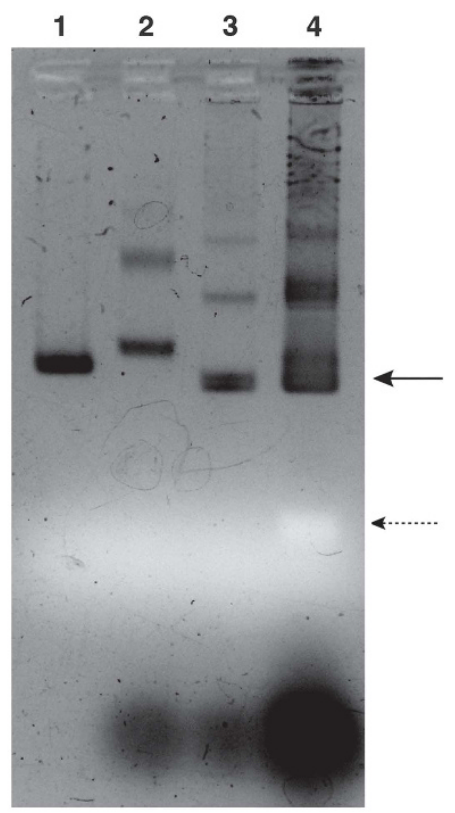

b
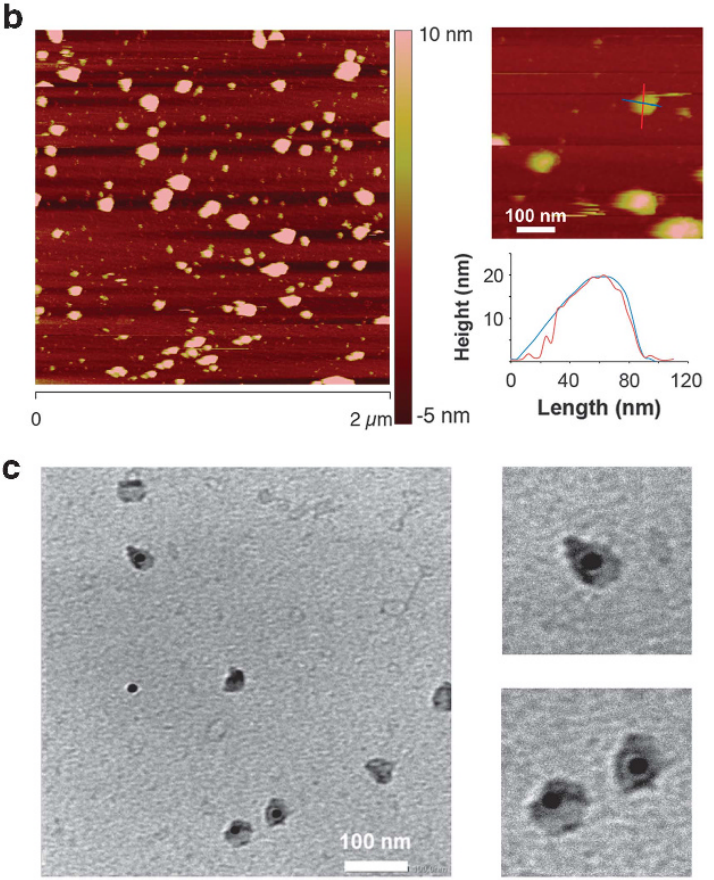
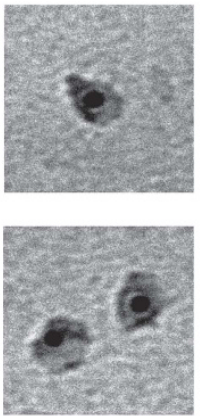

Figure 4 Selective encapsulation of a 10-nm gold nanoparticle (AuNP) in the DNA origami container. (a) Purification of the complexes using $1.5 \%$ agarose gel electrophoresis. Lane 1, M13 scaffold; lane 2, OM"; lane 3, closed motif (CM); lane 4, CM with AuNP. The band indicated by an arrow was collected. Bands in the above may correspond to complexes between DNA origami multimers (or OM") and AuNP. The band for unbound AuNPs is indicated by a dashed arrow. The dark band below is stained staple strands. (b) Atomic force microscope (AFM) analysis of the purified complexes. (c) Positively stained transmission electron microscope (TEM) images of the purified complexes. Enlarged images of $150 \times 150 \mathrm{~nm}$ area are shown on the right. OM, open motif.

plasmon resonance absorption of AuNPs, as indicated by an arrow in lane 4, far separated from the strong band of unbound AuNPs observed below (dashed arrow). This faint band co-migrated with the major band in lane 3, which corresponded to a CM as fluorescently visualized in DNA staining (Figure 4a, right). When control AuNPs, which contained non-complementary strands to those attached to the DNA origami containers, was used, no such band was observed above the unbound AuNPs (lane 4 in Supplementary Figure S2). Thus, the binding of AuNPs to DNA origami containers was completely sequence-selective. The absence of a band corresponding to the $\mathrm{OM}^{\prime \prime}$ (see lane 2) in lane 3 further confirmed the quantitative closing of the $\mathrm{OM}^{\prime \prime}$ into the $\mathrm{CM}$ via the $\mathrm{CM}^{\prime \prime}$. The colocalization of the AuNP absorbance and fluorescence signals in the band strongly suggested the existence of the desired CM-AuNP complexes. Thus, the band was excised from the gel, and the contents were extracted.

The purified band was first analyzed by AFM on mica in $1 \times \mathrm{TAE} /$ $\mathrm{Mg}^{2+}$ buffer solution (Figure 4b). A significantly larger number of spherical objects than those observed in Figure 3 were imaged. These spherical objects were classified by height into three groups: $8 \pm 3$, $15 \pm 5$ and $30 \pm 5 \mathrm{~nm}$. The proportion of each group was 42,31 and $27 \%$, respectively (for the counted numbers, see Supplementary Table S2). Considering that the 10-nm AuNPs were attached to the DNA origami container and the origami itself was $6-8 \mathrm{~nm}$ in dimension (slightly larger than the sum of two layers of 2-nm-thick DNA sheets), these groups of objects can be assigned to empty CMs $(8 \pm 3 \mathrm{~nm})$, desired CM-AuNP complexes stuck with a green face on the mica surface $(15 \pm 5 \mathrm{~nm}), \mathrm{CM}-\mathrm{AuNP}$ complexes stuck with one of the other small faces $(30 \pm 5 \mathrm{~nm})$ and/or dimers of the CM-AuNP complexes. The encapsulation yield of AuNP in a DNA origami container was estimated to be $30-50 \%$. This may be within a reasonable range considering that empty $\mathrm{CM}$ and CM-AuNP complexes were not separable under gel electrophoresis.

The transmission electron microscope imaging of the purified band clearly showed the successful formation of CM-AuNP complexes (Figure 4c). In accordance with the encapsulation yield estimated in the AFM analyses, empty CMs and many complexes containing exactly one AuNP in the stained DNA origami container were observed.

\section{CONCLUSION}

We successfully constructed a fusion nanomaterial of a self-assembled DNA nanostructure and an inorganic nanoparticle by selectively attaching a 10-nm AuNP to the interior of a DNA origami container. The attachment of the AuNP and the selective closure of the DNA origami container were fully controllable by adding appropriate DNA strands. The subsequent decoration of the exterior surface of the DNA origami container can be achieved by chemically modifying staple strands. In such a way, cell-specific ligands for targeted delivery or passivation polymers for stability enhancement in vivo can be attached. The attachment of other guests, such as enzymes or drugs, to the remaining interior faces is another attractive future target. ${ }^{28,29}$ In addition, the application of toehold-mediated, strand-exchange techniques should enable the programmed displaying and release of cargo. ${ }^{30,31}$ This may realize various sophisticated molecular systems, such as next-generation drug delivery systems.

\section{ACKNOWLEDGEMENTS}

This work was supported in part by a Grant-in-Aid for Scientific Research on Innovative Areas 'Fusion Materials' (25107734) from the Ministry of Education, Science, Sports, Culture and Technology, Japan (MEXT), and the MEXTSupported Program for the Strategic Research Foundation at Private 
Universities, 2009-2014, and was partially performed under the Cooperative Research Program of 'Network Joint Research Center for Materials and Devices' (for AK, YM, HM, KN and KI). Support from PRESTO, JST and from the Kansai University Subsidy for Supporting Young Scholars, 2012 is also acknowledged.

1 Kato, T., Sugawara, A. \& Hosoda, N. Calcium carbonate-organic hybrid materials. Adv. Mater. 14, 869-877 (2002).

2 Ruiz-Hitzky, E., Darder, M., Aranda, P. \& Ariga, K. Advances in biomimetic and nanostructured biohybrid materials. Adv. Mater. 22, 323-336 (2010).

3 Kuzuya, A. \& Ohya, Y. DNA nanostructures as scaffolds for metal nanoparticles. Polymer J. 44, 452-460 (2012).

4 Alivisatos, A. P., Johnsson, K. P., Peng, X., Wilson, T. E., Loweth, C. J., Bruchez, M. P. \& Schultz, P. G. Organization of 'nanocrystal molecules' using DNA. Nature 382, 609-611 (1996).

5 Mirkin, C. A., Letsinger, R. L., Mucic, R. C. \& Storhoff, J. J. A DNA-based method for rationally assembling nanoparticles into macroscopic materials. Nature $\mathbf{3 8 2}$ 607-609 (1996).

6 Wen, Y., McLaughlin, C. K., Lo, P. K., Yang, H. \& Sleiman, H. F. Stable gold nanoparticle conjugation to internal DNA positions: facile generation of discrete gold nanoparticle-DNA assemblies. Bioconjugate Chem. 21, 1413-1416 (2010).

7 Ohya, Y., Miyoshi, N, Hashizume, M., Tamaki, T., Uehara, T., Shingubara, S. \& Kuzuya, A. Formation of $1 \mathrm{D}$ and $2 \mathrm{D}$ gold nanoparticle arrays by divalent DNA-gold nanoparticle conjugates. Small 8, 2335-2340 (2012).

8 Seeman, N. Nucleic-acid junctions and lattices. J. Theor. Biol. 99, 237-247 (1982).

9 Rothemund, P. W. K. Folding DNA to create nanoscale shapes and patterns. Nature 440, 297-302 (2006)

10 Sharma, J., Chhabra, R., Andersen, C. S., Gothelf, K. V., Yan, H. \& Liu, Y. Toward reliable gold nanoparticle patterning on self-assembled DNA nanoscaffold. J. Am. Chem. Soc. 130, 7820-7821 (2008).

$11 \mathrm{Gu}$, H., Chao, J., Xiao, S.-J. \& Seeman, N. C. A proximity-based programmable DNA nanoscale assembly Line. Nature 465, 202-205 (2010).

12 Ding, B., Deng, Z., Yan, H., Cabrini, S., Zuckermann, R. N. \& Bokor, J. Gold Nanoparticle self-similar chain structure organized by DNA origami. J. Am. Chem. Soc. 132, 3248-3249 (2010).

13 Kuzuya, A., Koshi, N., Kimura, M., Numajiri, K., Yamazaki, T., Ohnishi, T., Okada, F. \& Komiyama, M. Programmed nanopatterning of organic/inorganic nanoparticles using nanometer-scale wells embedded in a DNA origami scaffold. Small 6, 2664-2667 (2010).

14 Endo, M., Yang, Y., Emura, T., Hidaka, K. \& Sugiyama, H. Programmed placement of gold nanoparticles onto a slit-type DNA origami scaffold. Chem. Commun. 47, 10743-10745 (2011)
15 Pal, S., Deng, Z., Wang, H., Zou, S., Liu, Y. \& Yan, H. DNA directed self-assembly of anisotropic plasmonic nanostructures. J. Am. Chem. Soc. 133, 17606-17609 (2011).

16 Pal S., Dutta P., Wang H., Deng Z., Zou S., Yan H. \& Liu Y. Quantum efficiency modification of organic fluorophores using gold nanoparticles on DNA origami scaffolds J. Phys. Chem. C 117, 12735-12744 (2013)

17 Zhao, Z., Jacovetty, E. L., Liu, Y. \& Yan, H. Encapsulation of gold nanoparticles in a DNA origami cage. Angew. Chem. Int. Ed. 50, 2041-2044 (2011).

18 Shen, X., Song, C., Wang, J., Shi, D., Wang, Z., Liu, N. \& Ding, B. rolling up gold nanoparticle-dressed DNA origami into three-dimensional plasmonic chiral nanostructures. J. Am. Chem. Soc. 134, 146-149 (2012).

19 Kuzyk, A., Schreiber, R., Fan, Z., Pardatscher, G., Roller, E.-M., Högele, A. Simmel, F. C., Govorov, A. O. \& Liedl, T. DNA-based self-assembly of chiral plasmonic nanostructures with tailored optical response. Nature 483, 311-314 (2013).

20 Schreiber, R., Do, J., Roller, E.-M., Zhang, T., Schüller, V. J., Nickels, P. C., Feldmann, J. \& Liedl, T. Hierarchical assembly of metal nanoparticles,quantum dots and organic dyes using DNA origami scaffolds. Nat. Nanotechnol. 9, 74-78 (2014).

21 Kuzuya, A. \& Komiyama, M. Design and construction of a box-shaped 3D-DNA origami. Chem. Commun. 4182-4184 (2009).

22 Andersen, E. S., Dong, M., Nielsen, M. M., Jahn, K., Subramani, R., Mamdouh, W., Golas, M. M., Sander, B., Stark, H., Oliveira, C. L. P., Pedersen, J. S., Birkedal, V., Besenbacher, F., Gothelf, K. V. \& Kjems, J. Self-assembly of a nanoscale DNA box with a controllable lid. Nature 459, 73-76 (2009).

23 Zadegan, R. M., Jepsen, M. D. E., Thomsen, K. E., Okholm, A. H., Schaffert, D. H., Andersen, E. S., Birkedal, V. \& Kjems, J. Construction of a 4 zeptoliters switchable 3D DNA box origami. ACS Nano 6, 10050-10053 (2012).

24 Ke, Y., Sharma, J., Liu, M., Jahn, K., Liu, Y. \& Yan, H. Scaffolded DNA origami of a DNA tetrahedron molecular container. Nano Lett. 9, 2445-2447 (2009).

25 Endo, M., Hidaka, K., Kato, T., Namba, K. \& Sugiyama, H. DNA prism structures constructed by folding of multiple rectangular arms. J. Am. Chem. Soc. 131, 15570-15571 (2009).

26 Kuzuya, A. \& Ohya, Y. Nanomechanical molecular devices made of DNA origami. Acc. Chem. Res. 47, 1742-1749 (2014).

27 Loweth, C., Caldwell, W., Peng, X., Alivisatos, A. P. \& Schultz, P. G. DNA-based assembly of gold nanocrystals. Angew. Chem. Int. Ed. 38, 1808-1812 (1999).

28 Numajiri, K., Yamazaki, T., Kimura, M., Kuzuya, A. \& Komiyama, M. Discrete and active enzyme nanoarrays on DNA origami scaffolds purified by affinity tag separation. J. Am. Chem. Soc. 132, 9937-9939 (2010)

29 Yamazaki, T., Heddle, J. G., Kuzuya, A. \& Komiyama, M. Orthogonal enzyme arrays on a DNA origami scaffold bearing size-tunable wells. Nanoscale 6, 9122-9126 (2014)

30 Yurke, B., Turberfield, A. J., Mills, A. P., Simmel, F. C. \& Neumann, J. L. A DNA-fuelled molecular machine made of DNA. Nature 406, 605-608 (2000).

31 Douglas, S. M., Bachelet, I. \& Church, G. M. A logic-gated nanorobot for targeted transport of molecular payloads. Science 335, 831-834 (2012).

Supplementary Information accompanies the paper on Polymer Journal website (http://www.nature.com/pj) 